\title{
WEAKLY TOPOLOGIZED ALGEBRAS
}

\section{SETH WARNER}

Let $E$ be an algebra over the reals or complex numbers, $E^{\prime}$ a total subspace of the algebraic dual of $E, \sigma\left(E, E^{\prime}\right)$ the weak topology defined on $E$ by $E^{\prime}$. In Theorems 1 and 2 of [2], the author established necessary and sufficient conditions for $E$, equipped with topology $\sigma\left(E, E^{\prime}\right)$, to be a locally $m$-convex algebra and to be a topological algebra. Actually, these conditions may be combined as follows:

Theorem 1. The following are equivalent: (1) $E ; \sigma\left(E, E^{\prime}\right)$ is a locally $m$-convex algebra. (2) $E ; \sigma\left(E, E^{\prime}\right)$ is a topological algebra. (3) For every $g \in E^{\prime}$, the kernel of $g$ contains a (weakly) closed ideal of finite codimension. (4) $(x, y) \rightarrow x y$ is (weakly) continuous at $(0,0)$.

Proof. (1) and (3) are equivalent by Theorem 1 of [2]; (2) and (4) are equivalent by Proposition 6 of $[1$, p. 8] since $(x, y) \rightarrow x y$ is bilinear; and clearly (1) implies (2). It remains to show (4) implies (3). For this we need the following lemma, the proof of which is very similar to that of Lemma 1 of [2] and is therefore omitted:

Lemma. Let $g \in E^{\prime}$, let $W$ be a neighborhood of zero for $\sigma\left(E, E^{\prime}\right)$, and let $J$ be a subspace of $E$ such that $J \subseteq W \subseteq W \cup W^{2} \cup W^{3} \subseteq[x|| g(x) \mid$ $\leq 1]$. Then $J, J E, E J$, and EJE are contained in the kernel of $g$.

To complete the proof of the theorem, consider any $g \in E^{\prime}$. There exists by (4) a (weakly) closed, convex, equilibrated neighborhood $W$ of 0 such that $W \subseteq W \cup W^{2} \cup W^{3} \subseteq[x|| g(x) \mid \leq 1]$. Let $J=\cap\left[u^{-1}(0) \mid u\right.$ $\left.\in W^{0}\right]$. Then $J \subseteq W^{00}=W$, and $J$ is a (weakly) closed subspace of finite codimension by Lemma 2 of [2]. The ideal $L$ generated by $J$ is clearly identical with the subspace generated by $J \cup E J \cup J E \cup E J E$ and hence by the lemma is contained in the kernel of $g$. Let $\phi$ be the canonical map from locally convex space $E$ onto the Hausdorff, finite-dimensional, locally convex space $E / J . \phi(L)$ is a subspace of finite-dimensional $E / J$ and hence is closed. Thus $L=L+J=\phi^{-1}(\phi(L))$ is (weakly) closed in $E$. Therefore $L$ is a (weakly) closed ideal of finite codimension contained in the kernel of $g$, and the proof is complete.

Now let $E$ be a Hausdorff locally $m$-convex algebra, $E^{\prime}$ its topological dual. Multiplication is then separately continuous in each variable

Received by the editors April 27, 1956. 
when $E$ is given the associated weak topology $\sigma\left(E, E^{\prime}\right.$ ). (For if $L_{a}: x \rightarrow a x$, to show $L_{a}$ is continuous when $E$ is equipped with $\sigma\left(E, E^{\prime}\right)$, it suffices to show that for all $u \in E^{\prime}$, the linear form $u \circ L_{a}$ is weakly continuous; but $u \circ L_{a}$ is a continuous linear form on $E$ equipped with its given topology; hence $u \circ L_{a} \in E^{\prime}$ and so is weakly continuous.) The following theorem shows, however, that for a familiar class of Banach algebras, multiplication is not weakly continuous in both variables.

THEOREM 2. Let E be a commutative, semi-simple, self-adjoint Banach algebra over the complex numbers. Then $E ; \sigma\left(E, E^{\prime}\right)$ is a topological algebra if and only if $E$ is finite-dimensional.

Proof. The condition is clearly sufficient, for if $E$ is finite-dimensional, $\sigma\left(E, E^{\prime}\right)$ is the given topology of $E$. Necessity: Let $\mathfrak{T}(E)$ be the set of all (continuous) nonzero multiplicative linear forms. Then by hypothesis $\{0\}=\bigcap\left[u^{-1}(0) \mid u \in \mathfrak{N}(E)\right]$. Suppose $E$ is infinitedimensional. Then $\{0\}$ has infinite codimension, and hence $\mathfrak{T}(E)$ must be infinite. Let $\left\{u_{n}\right\}_{n=1}^{\infty}$ be a sequence of distinct members of $\mathfrak{T}(E)$. It is well-known that $\mathfrak{T}(E)$ is a bound subset of the strong dual $E_{b}^{\prime}$ of $E$ and that $E_{b}^{\prime}$ is complete; hence $g=\sum_{n=1}^{\infty} 2^{-n} u_{n}$ exists and is a member of $E^{\prime}$. By (3) of Theorem 1, the kernel of $g$ contains a weakly closed ideal $L$ of finite codimension. For any $x \in L, x x^{*} \in L$, whence $0=g\left(x x^{*}\right)=\sum_{n=1}^{\infty} 2^{-n} u_{n}\left(x x^{*}\right)=\sum_{n=1}^{\infty} 2^{-n}\left|u_{n}(x)\right|^{2}$; therefore $u_{n}(x)=0$ for all $n$ and all $x \in L$, i.e., $u_{n}(L)=\{0\}$ for all $n$. Therefore there exists, for each integer $n$, a nonzero multiplicative linear form $\bar{u}_{n}$ on the finite-dimensional algebra $E / L$ satisfying $\bar{u}_{n} \circ \phi=u_{n}$, where $\phi$ is the canonical homomorphism from $E$ onto $E / L .\left\{\bar{u}_{n}\right\}_{n=1}^{\infty}$ is then a sequence of distinct, nonzero multiplicative linear forms on $E / L$ and thus by Proposition 2 of [2] is a linearly independent subset of the dual $(E / L)^{\prime}$ of $E / L$. Hence $(E / L)^{\prime}$ is infinite-dimensional. But as $L$ is of finite codimension, $E / L$ and hence also $(E / L)^{\prime}$ are finitedimensional. This contradiction completes the proof.

The proof of Theorem 2 is available under far more general circumstances than those of the theorem. Indeed, an obvious modification of the proof shows that if $E$ is a locally $m$-convex algebra satisfying the following conditions, multiplication is not continuous for the associated weak topology: (1) $\{0\}=\bigcap\left[u^{-1}(0) \mid u \in \mathfrak{N}(E)\right]$, where $\mathfrak{T}(E)$ is the set of all continuous, nonzero multiplicative linear forms on $E$; (2) $E$ is self-adjoint if the field of scalars is the complex numbers; (3) There exists an infinite subset $\left\{u_{n}\right\}_{n=1}^{\infty}$ of $\mathscr{N}(E)$ and scalars $\lambda_{n}>0$ such that $\left\{\lambda_{n} u_{n}\right\}_{n=1}^{\infty}$ is a bound subset of the strong dual $E_{b}^{\prime}$ of $E$; (4) $E_{b}^{\prime}$ is quasi-complete. 


\section{REFERENCES}

1. N. Bourbaki, Espaces vectoriels topologiques, Chaps. 1-2 Actualités Scientifiques et Industrielles no. 1189, Paris, Hermann.

2. Seth Warner, Weak locally multiplicatively-convex algebras, Pacific Journal of Mathematics vol. 5 (1955) pp. 1025-1032.

DUKE UNIVERSITY

\section{SUMS OF THREE SQUARES}

N. C. ANKENY 1

Introduction. I would like to present here a short and elementary proof of the following theorem.

THEOREM 1. If $m$ is a positive integer not of the form $4^{a}(8 n+7)$, then $m$ is the sum of three squares.

We make use of an elegant method of Professor H. Davenport [1] in the Geometry of Numbers.

Without loss of generality we will prove Theorem 1 only when $m$ is square free. (In the following $m$ will be assumed to be square free.) In $\$ 1$ we shall prove Theorem 1 when $m \equiv 3(\bmod 8)$. In $\$ 2$ we will merely outline the proof when $m \equiv 1,2,5,6(\bmod 8)$, as the proof is almost identical except for minor changes.

We shall only assume the reader is familiar with the elementary facts of the law of quadratic reciprocity, Minkowski's Theorem on lattice points contained within convex symmetric bodies; and when a positive integer is the sum of two squares.

1 . Let $m$ be a positive square free integer $\equiv 3(\bmod 8)$, and $m=p_{1} p_{2} \cdots p_{r}$ where $p_{j}^{\prime}$ s are primes.

Denote by $q$ a positive prime which satisfies

$$
\begin{aligned}
\left(-2 q / p_{j}\right) & =+1, \quad j=1,2, \cdots, r, \\
q & \equiv 1(\bmod 4)
\end{aligned}
$$

with $(a / b)$ denoting the Jacobi Symbol. We see that such a prime exists by Dirichlet's theorem regarding primes in an arithmetic pro-

Received by the editors April 26. 1956.

1 This research was supported by the United States Air Force through the Air Force Office of Scientific Research of the Air Research and Development Command, under contract No. AF 18 (603)-90. Reproduction in whole or in part is permitted for any purpose of the United States Government. 